\title{
HELIOS-CR - A 1-D Radiation-Magnetohydrodynamics Code with Inline Atomic Kinetics Modeling
}

\author{
J. J. MacFarlane, I. E. Golovkin, and P. R. Woodruff
}

\author{
Prism Computational Sciences \\ 455 Science Drive, Suite 140 \\ Madison, WI 53711
}




\begin{abstract}
HELIOS-CR is a user-oriented 1-D radiation-magnetohydrodynamics code to simulate the dynamic evolution of laser-produced plasmas and z-pinch plasmas. It includes an in-line collisionalradiative (CR) model for computing non-LTE atomic level populations at each time step of the hydrodynamics simulation. HELIOS-CR has been designed for ease of use, and is well-suited for experimentalists, as well as graduate and undergraduate student researchers. The energy equations employed include models for laser energy deposition, radiation from external sources, and high-current discharges. Radiative transport can be calculated using either a multi-frequency flux-limited diffusion model, or a multi-frequency, multi-angle short characteristics model. HELIOS-CR supports the use of SESAME equation of state (EOS) tables, PROPACEOS EOS/multi-group opacity data tables, and nonLTE plasma properties computed using the inline CR modeling. Time-, space-, and frequency-dependent results from HELIOS-CR calculations are readily displayed with the HydroPLOT graphics tool. In addition, the results of HELIOS simulations can be post-processed using the SPECT3D Imaging and Spectral Analysis Suite to generate images and spectra that can be directly compared with experimental measurements. The HELIOS-CR package runs on Windows, Linux, and Mac OSX platforms, and includes online documentation. We will discuss the major features of HELIOS-CR, and present example results from simulations.
\end{abstract}

Keywords: Hydrodynamics, radiation transport, atomic kinetics, laser-produced plasmas, z-pinch plasmas, high energy density physics. 


\section{INTRODUCTION}

Simulations of the dynamics of plasmas created in high energy density plasma physics experiments play a crucial role in analyzing and interpreting experimental measurements. Radiationhydrodynamics codes are often used to study the dynamics of laser-produced, radiatively-heated, and high-current z-pinch plasmas created for the study of inertial confinement fusion, as well as the study of astrophysics and industrial applications [1,2]. HELIOS-CR is a 1-D radiation-magnetohydrodynamics code that is used to simulate the dynamic evolution of plasmas created in high energy density physics (HEDP) experiments. In designing HELIOS-CR, a substantial emphasis was placed on making it easy to use, so that it could be used not only by researchers experienced in the fields of radiation-hydrodynamics and HEDP plasmas, but also by graduate and undergraduate students being trained in the physical sciences.

HELIOS-CR solves Lagrangian hydrodynamics equations in planar, cylindrical, and spherical geometries. Plasmas may be composed of a single material or multiple layers (or regions) of materials. HELIOS-CR supports the utilization of equation of state and opacity databases that are generated under the assumption of local thermodynamic equilibrium (LTE), as well as those generated for non-LTE

plasmas. HELIOS-CR provides the added capability to simulate the non-LTE kinetics of plasmas by solving multi-level atomic rate equations at each time step in the simulation. This can be particularly important when modeling experiments in which deviations from LTE can significantly affect the overall energetics of the plasma, such as when radiation energy losses represent a significant fraction of the overall energy budget.

HELIOS-CR includes a graphical user interface for setting up problems, online documentation, a graphical progress monitor, and the HydroPLOT graphics package for viewing space-, time-, and frequency-dependent results. HELIOS-CR also conveniently interfaces with other HEDP simulation tools used in simulating experiments. The VISRAD radiation view factor code [3] includes the capability to generate time- and frequency-dependent external radiation fields that are used as a radiation boundary condition for HELIOS-CR. In addition, the time-dependent plasma distributions computed using 
HELIOS-CR can be post-processed using the SPECT3D Imaging and Spectral Analysis Suite [4] to generate images and spectra (which include instrumental effects) that can be directly compared with experimental measurements. Figure 1 shows a schematic illustration of how HELIOS-CR interfaces with other codes and data.

We describe some of the major features of HELIOS-CR, and present some sample results. A description of the PROPACEOS equation of state and opacity database used by HELIOS-CR is provided in the appendix.

\section{MAJOR FEATURES OF HELIOS-CR}

HELIOS is a 1-D Lagrangian radiation-magnetohydrodynamics code designed to simulate the evolution of a wide variety of high energy density plasmas. HELIOS-CR is a version of HELIOS that includes the capability to perform inline non-LTE atomic kinetics (i.e., collisional-radiative) calculations at each time step in the hydrodynamics simulation.

HELIOS-CR solves the equation of motion for a single fluid. Electrons and ions are assumed to be co-moving. Pressure contributions to the equation of motion come from electrons, ions, radiation, and the magnetic field. Energy transport in the plasma can be treated using either a one-temperature $\left(T_{i}=T_{e}\right)$ or two-temperature $\left(T_{i} \neq T_{e}\right)$ model. Both the electrons and ions are assumed to have Maxwellian distributions defined by their respective temperatures, $T_{i}$ and $T_{e}$. Options for thermal conduction models include: Spitzer conductivities, uniform (user-specified) material-dependent conductivities, and a hybrid Spitzer-uniform model.

Material EOS properties are based on either SESAME tables [5] or PROPACEOS tables. Opacities are based either on tabulated multi-group (i.e., frequency binned) PROPACEOS data, or, in the case when inline CR modeling is used, frequency-dependent opacities based on non-LTE atomic level populations. In the latter case, an adaptive frequency mesh is used. A brief summary of the models used in PROPACEOS is provided in Appendix A. Radiation emission and absorption terms are coupled to the electron temperature equation. Multi-frequency radiation intensities are computed using either a fluxlimited radiation diffusion model, or a multi-angle model based on the method of short characteristics (the multi-angle model is currently restricted to planar geometry). 
Laser energy deposition is computed using an inverse Bremsstrahlung model, with the restriction that no energy in the beam passes beyond the critical surface. In planar geometry, laser light is transported along a single ray with incidence angle $\theta$. In spherical geometry, a multi-ray, conical beam model is used. Laser deposition in cylindrical geometry is not currently supported, but is expected to be added in the future.

A magnetic diffusion model has recently been added to HELIOS-CR for calculations in cylindrical geometry. This provides the capability to simulate z-pinch plasmas created by high-current discharges.

\section{Conservation Equations}

In Lagrangian hydrodynamics, the spatial grid moves with the fluid. No mass crosses volume element boundaries. It is useful to express the spatial coordinate in terms of the independent Lagrangian mass variable:

$$
d m_{0}=\rho(r) r^{\delta-1} d r
$$

where $\rho$ is the mass density, $r$ is the spatial position (i.e., the radius in cylindrical and spherical geometries), and $\delta=1,2$, or 3 for planar, cylindrical, or spherical geometry, respectively. In this system, the continuity (mass conservation) equation is automatically satisfied.

The momentum conservation equation is solved in the one-fluid approximation, where the plasma electrons and ions are assumed to flow together as a single fluid. The momentum conservation equation is given by:

$$
\frac{\partial u}{\partial t}=-r^{\delta-1} \frac{\partial}{\partial m_{0}}(P+q)-\frac{1}{8 \pi r} \frac{\partial}{\partial m_{0}}\left(r^{2} B^{2}\right)
$$

where $u$ is the fluid velocity, $t$ is the time, $P$ is the total pressure due to electrons, ions, and radiation, $q$ is the von Neumann artificial viscosity [6], and $B$ is the magnetic field induced by the axial current (nonzero for cylindrical geometry only). Specifically, $B$ is the azimuthal component of the magnetic induction vector (i.e., $B=\overrightarrow{B_{\theta}}(r, t)$ ). The artificial viscosity, which is included in the equation of motion to handle shocks, effectively smoothes the shock over a small number of zones. 
The conservation of energy equations are written in terms of temperature diffusion equations for the electrons and ions, and are given by:

$$
C_{\mathrm{v}, e} \frac{\partial T_{e}}{\partial t}=\frac{\partial}{\partial m_{0}}\left(r^{\delta-1} \xi_{e} \frac{\partial T_{e}}{\partial r}\right)-\omega_{e i}\left(T_{e}-T_{i}\right)-\left[\frac{\partial E_{e}}{\partial V}+P_{e}\right] \frac{\partial V}{\partial t}+R_{A b s}-R_{E m i s}+\Psi+S_{e}
$$

and

$$
C_{\mathrm{v}, i} \frac{\partial T_{i}}{\partial t}=\frac{\partial}{\partial m_{0}}\left(r^{\delta-1} \xi_{i} \frac{\partial T_{i}}{\partial r}\right)+\omega_{e i}\left(T_{e}-T_{i}\right)-\left[\frac{\partial E_{i}}{\partial V}+P_{i}\right] \frac{\partial V}{\partial t}-q \frac{\partial V}{\partial t}
$$

where $T_{e}\left(T_{i}\right)$ is the electron (ion) temperature, $C_{\mathrm{v}, e}\left(C_{\mathrm{v}, i}\right)$ is the electron (ion) specific heat, $\xi_{e}\left(\xi_{i}\right)$ is the electron (ion) thermal conductivity, $E_{e}\left(E_{i}\right)$ is the electron (ion) specific internal energy, $\omega_{e i}$ is the electron-ion collisional coupling term, $S_{e}$ is the source term due to laser energy deposition, $\Psi$ is the joule heating term (for MHD option), and $R_{A b s}$ and $R_{E m i s}$ are the radiation absorption and emission terms.

The radiative emission and absorption terms are given by:

$$
R_{\text {Emis }}=\frac{8 \pi\left(k T_{e}\right)^{4}}{c^{2} h^{3}} \sum_{g}^{N_{F}}\left(\sigma_{g}^{P E} \int_{x_{g}}^{x_{g+1}} d x \frac{x^{3}}{e^{x}-1}\right), \quad x=\frac{h v}{k T_{e}}
$$

and

$$
R_{A b s}=c \sum_{g}^{N_{F}}\left(\sigma_{g}^{P A} E_{R, g}\right)
$$

where $g$ is the frequency group index, $N_{F}$ is the number of frequency groups, $h$ is Planck's constant, $E_{R, g}$ is the radiation energy density for group $g$, and $\sigma_{g}^{P E}$ and $\sigma_{g}^{P A}$ are the Planck mean opacities for group g for emission and absorption, respectively. For opacity tables generated under the assumption of LTE, $\sigma_{g}^{P E}$ and $\sigma_{g}^{P A}$ are equal, as Kirchoff's Law $\left(\eta_{v}=\kappa_{v} B_{v}\right)$ is valid.

The thermal conduction coefficients for electrons and ions in Eqs. (3) and (4) are based on Spitzer conductivities, and can be written as:

$$
\xi_{\mathrm{e}, i}=20\left(\frac{2}{\pi}\right)^{3 / 2} \frac{\left(k T_{e, i}\right)^{5 / 2} k \tau_{e, i}}{m_{e}^{1 / 2} e^{4} Z \ln \Lambda_{e i, i i}}
$$

where $\Lambda_{e i}$ and $\Lambda_{i i}$ are the Coulomb terms for electron-ion and ion-ion collisions, respectively, $\tau_{i}=1$, and

$$
\tau_{e}=\frac{0.43 Z}{3.44+Z+0.26 \ln Z}
$$


where $Z$ is the mean charge. The thermal electron-ion coupling coefficient is given by:

$$
\omega_{e i}=C_{v, e}\left(\frac{Z}{A}\right)^{2} \frac{\ln \Lambda_{e i}}{V T_{e}^{3 / 2}}
$$

where $A$ is the mean atomic weight.

\section{Magnetic Diffusion Model}

HELIOS-CR includes an MHD model for cylindrical geometry. The magnetic diffusion equation in cylindrical geometry is given by:

$$
\frac{1}{c \rho r^{2}} \frac{\partial G}{\partial t}=\frac{c}{4 \pi} \frac{\partial}{\partial m_{o}}\left(\frac{\eta}{r} \frac{\partial G}{\partial r}\right)-\frac{G}{c} \frac{\partial}{\partial m_{o}}\left(\frac{u}{r}\right)
$$

where $G(r, t)=r B(r, t), c$ is the speed of light, and $\eta$ is the electrical resistivity. The value of $G$ at the boundary is constrained by the Biot-Savart law:

$$
G\left(r=R_{\max }, t\right)=\frac{2}{C} I_{D}(t)
$$

where $R_{\max }$ is the outer radius of the cylindrical grid, and $I_{D}(t)$ is the discharge current at time $t$.

The electrical resistivity model is based on the classical transport theory and includes the effects of Coulomb collisions and electron-ion collisions. This can be written as [7]:

$$
\eta=C_{\eta} Z T_{i}^{-3 / 2} \ln \Lambda_{e i}
$$

where $C_{\eta}=5.80 \times 10^{-15} \mathrm{sec}^{-3 / 2}$. For low degrees of ionization $\left(\bar{Z} \leq 10^{-2}\right)$, the resistivity is given by the weakly ionized form:

$$
\eta=\frac{m_{e}^{1 / 2}}{e^{2}} T_{i}^{1 / 2} \frac{\sigma_{e a}}{Z}
$$

where $\sigma_{e a}$ is the electron-atom collisional cross section. For , the resistivity is obtained by interpolating between values obtained from Eqs. (12a) and (12b).

\section{$\underline{\text { Radiation Modeling }}$}

Radiation transport can be calculated using either a diffusion transport model (all geometries) or a multi-angle transport model (planar geometry only). When using multi-group (tabulated) opacities, the transport equation is evaluated for each frequency group using Planck and Rosseland group-averaged opacities. When the collisional-radiative (CR) modeling is employed, a frequency grid is set up that 
resolves the bound-bound profiles and bound-free edges. In this case, the transport equation is evaluated at each discrete frequency point.

In multi-group calculations, users can specify the number of frequency groups in the calculation, as well as the frequency grid parameters in the calculation. Because HELIOS-CR supports the ability to regroup opacities, new multi-group opacity datasets are not required.

\section{Flux-Limited Diffusion Radiation Transport Model}

The radiation transport equation for the flux-limited diffusion model can be written as:

$$
V \frac{d E_{R, g}}{d t}=\frac{\partial}{\partial m_{0}}\left(r^{\delta-1} \frac{c V}{3 \sigma_{g}^{R}} \frac{\partial E_{R, g}}{\partial r}\right)-\frac{4}{3} E_{R, g} \frac{\partial V}{\partial t}+R_{E m i s, g}-R_{A b s, g}
$$

where $E_{R, g}$ is the radiation energy density at frequency group $g, V$ is the specific volume $\left(=\rho^{-1}\right), \sigma_{g}^{R}$ is

the Rosseland opacity for frequency group $g$, and $R_{E m i s, g}$ and $R_{A b s, g}$ are the radiative emission and absorption terms for a single group $g$ (see Eqs. (5) and (6)). When CR modeling is used, the radiation energy density is solved at discrete frequency points, and both the Rosseland and Planck-averaged group opacities are equal to $V \kappa_{v}$, where $\kappa_{v}$ is the absorption coefficient.

HELIOS-CR has been set up to use one of two different flux limiters when the diffusion model is employed. These include the Larsen flux limiter [8], and the Levermore-Pomraning limiter [9]. By default, the Larsen limiter is used.

\section{Multi-angle Radiation Transport Model}

HELIOS-CR includes the option of using a multi-angle radiative transfer model for simulations with planar geometry. This model, which is based on the work of Olson and Kunasz [10], solves the timeindependent form of the transfer equation. The formal solution to the transfer equation in planar geometry can be written as [11]:

$$
I^{+}\left(\tau_{v}, \mu, v\right)=I^{+}\left(T_{v}, \mu, v\right) e^{\left[-\left(T_{v}-\tau_{v}\right) / \mu\right]}+\mu^{-1} \int_{\tau_{v}}^{T_{v}} d t S_{v}(t) e^{\left[-\left(t-\tau_{v}\right) / \mu\right]}
$$

where $I^{+}\left(\tau_{v}, \mu, v\right)$ is the specific intensity in the "+" direction $(0 \leq \mu \leq 1)$ at frequency $v$, at a position given by optical depth $\tau_{v}$, and along a ray defined by the cosine angle $\mu=\cos \theta$ ( $\theta=$ angle with respect 
to the surface normal). $\tau_{v}$ is the frequency-dependent optical depth measured along a path normal to the slab boundary $\left(0 \leq \tau_{v} \leq T_{v}\right)$, and $S_{v}$ is the source function. In the "-" direction $(-1 \leq \mu \leq 0)$, the specific intensity is:

$$
I^{-}\left(\tau_{v}, \mu, v\right)=I^{-}(0, \mu, v) e^{\left[-\tau_{v} / \mu\right]}+\mu^{-1} \int_{0}^{\tau_{v}} d t S_{v}(t) e^{\left[-\left(\tau_{v}-t\right) / \mu\right]}
$$

The flux at a given position is computed from the angle-average of the specific intensities:

$$
F\left(\tau_{v}\right)=2 \pi \int_{-1}^{1} I\left(\tau_{v}, \mu, v\right) \mu d \mu
$$

Eqs. (14) and (15) are solved on a discretized optical depth grid. In the case of external radiation fields, non-zero boundary conditions are applied at $\tau_{v}=0$ and $\tau_{v}=T_{v}$.

\section{Atomic Kinetics Model}

When using inline collisional-radiative modeling within HELIOS-CR, non-LTE atomic level populations are updated by solving a coupled set of atomic rate equations at each time step in the simulation. The rate equation for atomic level $i$ can be written as:

$$
\frac{d n_{i}}{d t}=-n_{i} \sum_{i \neq j}^{N_{L}} W_{i j}+\sum_{i \neq j}^{N_{L}} n_{j} W_{j i}
$$

where $W_{i j}$ and $W_{j i}$ represent the depopulating and populating rates between levels $i$ and $j, n_{i}$ is the number density of level $i$, and $N_{L}$ is the total number of levels in the system. For upward transitions $(i<j)$,

$$
\begin{aligned}
& / \quad n_{e} C_{i j}+B_{i j} \overline{J_{i j}} \quad \text { (excitations) } \\
& W_{i j}=\langle \\
& \backslash \quad n_{e} \gamma_{i j}+\beta_{i j}+\Omega_{i j} \quad \text { (ionizations) }
\end{aligned}
$$

while for downward transitions $(i>j)$,

$$
\begin{aligned}
& / \quad n_{e} D_{j i}+A_{j i}+B_{j i} \overline{J_{i j}} \quad \text { (deexcitations) } \\
& W_{j i}=\langle \\
& \backslash \quad n_{e}^{2} \delta_{j i}+n_{e}\left(\alpha_{j i}^{R R}+\alpha_{j i}^{D R}\right) \quad \text { (recombinations) }
\end{aligned}
$$


where $n_{e}$ is the electron density; $\overline{J_{i j}}$ is the frequency-averaged mean intensity of the radiation field over a line profile; $C_{i j}, \gamma_{i j}, D_{j i}$, and $\delta_{j i}$ are rate coefficients for collisional excitation, ionization, deexcitation, and recombination; $A_{j i}, B_{i j}$, and $B_{j i}$ are Einstein coefficients for spontaneous emission, and stimulated absorption and emission; $\beta_{i j}$ is the photoionization rate; $\Omega_{i j}$ is the autoionization rate; $\alpha_{j i}^{R R}$ is the radiative recombination rate coefficient; and $\alpha_{j i}^{D R}$ is the dielectronic recombination rate coefficient (or, in the case of treating dielectronic recombination using explicit autoionization levels, the electron capture rate coefficient). In calculating photoexcitation and photoionization rates, frequency- and spatiallydependent mean intensities, $J_{v}(r)$, are used.

Continuum lowering effects are modeled using an occupation probability model [12], supplemented by the ionization potential depression formalism of More [13]. The occupation probability model produces a continuous reduction in the effective statistical weights of energy levels with increasing density, so that the relatively high- $n$ states ( $n=$ principal quantum number) cannot be populated at high densities. This occupation probability formalism compares favorably with results from ion microfield calculations of argon at high densities [14] using the APEX code [15]. The ionization energy thresholds are depressed using the More model, which results in an enhancement of ionization rates and a shift in the location of bound-free edges in computed spectra.

Atomic cross section data are generated using the ATBASE suite of codes [16]. Energy levels, photoionization cross sections, oscillator strengths, autoionization rates, and energy levels are calculated using a configuration interaction model with Hartree-Fock wavefunctions. Collisional coupling between states is complete - i.e., all thermal (non-autoionizing) and autoionizing states are collisionally coupled with electron-impact collisional excitation and ionization cross sections computed using a distorted wave model. Dielectronic recombination processes involving autoionization states of Ne-like ions and higher are treated explicitly, with electron capture rates determined from detailed balance with their corresponding autoionization rates. For lower ionization stages, autoionization states are not explicitly included in the atomic model, and effective dielectronic recombination rates are utilized.

In HELIOS-CR calculations, it is possible to use conventional radiation modeling (e.g., multigroup diffusion with LTE opacities) until a user-specified electron temperature threshold is reached. 
The purpose of this is to reduce computational time requirements, and this approach is often justified by the fact that radiation losses from the plasma do not become significant until relatively high temperatures are achieved. When doing this, it is advisable to perform test calculations in which the non-LTE atomic kinetics modeling is turned on at lower temperatures to check the sensitivity to the threshold.

HELIOS-CR is currently capable of performing non-LTE atomic kinetics calculations with up to $10^{3}$ discrete atomic energy levels. Atomic models - i.e., a selected set of atomic energy levels and a specification of how the levels are split (e.g., configuration averaged, L-S term split, or fine structure split) - can be chosen from a collection of default models, or users can generate their own customized atomic models. To facilitate the generation of customized atomic models, the AtomicModelBuilder application was developed to conveniently allow users to select energy levels from the atomic data library and to specify the degree of level splitting.

\section{$\underline{\text { External Radiation Sources }}$}

The radiative heating of plasmas due to external radiation fields can be simulated using either: (i) a time-dependent single radiation temperature model (in which both the frequency-dependence of the radiation field and the flux are specified using a “drive” temperature $\left(T_{R}(t)\right)$; or (ii) a time- and frequency-dependent non-Planckian radiation field calculated using the VISRAD view factor code [3]. In the latter case, VISRAD code generates a data file in a format that can be read by HELIOS-CR.

\section{Laser Deposition Model}

The laser deposition model in HELIOS-CR utilizes ray tracing algorithms for spherical and planar geometries. It is assumed that laser light propagates through the plasma instantaneously. Effects due to the polarization of laser light are currently neglected. User input to HELIOS-CR includes the laser wavelength $\left(\lambda_{L}\right)$, the time-dependent incident laser power $\left(P_{L}(t)\right)$, and the boundary at which the incident laser source originates. 
Laser energy is deposited in the plasma using an inverse Bremsstrahlung model when the electron density is less that the critical density. For laser light with a wavelength $\lambda_{L}$, the critical density is given by [17]:

$$
n_{e, c r i t}=\frac{\varepsilon_{0} m_{e}}{e^{2}} \omega_{L}^{2}=\left(1.11 \times 10^{21} \mathrm{~cm}^{-3}\right) \lambda_{L, \mu m}^{-2}
$$

where $\varepsilon_{0}$ is the permittivity in free space, $\omega_{L}=2 \pi c / \lambda_{L}$ is the angular frequency of the laser light, $c$ is the speed of light, $\lambda_{L, \mu m}$ is the laser wavelength in $\mu \mathrm{m}$, and $m_{e}$ and $e$ are the electron mass and charge, respectively. Laser energy is not allowed to penetrate beyond the critical surface.

The depth at which the laser light penetrates is determined from the absorption coefficient [18]:

$$
\kappa=(2 \pi)^{1 / 2}\left(\frac{16 \pi}{3}\right) \frac{e^{6}}{c\left(m_{e} k T_{e}\right)^{3 / 2}} Z n_{e}^{2} \frac{\ln \Lambda}{\omega_{L}^{2} \sqrt{1-\left(\omega_{P} / \omega_{L}\right)^{2}}}
$$

where $k$ is Boltzmann's constant, $n_{e}$ is the electron density, $T_{e}$ is the electron temperature, $Z$ is the mean charge of the plasma, $\omega_{P}$ is the plasma frequency, and $\ln \Lambda$ is the Coulomb logarithm.

In planar geometry, laser deposition is computed by solving an integral form of the radiative transfer equation along a single ray which has an angle of incidence $\theta$ with respect to the surface normal. In spherical geometry, the beam is modeled with multiple rays contained within a cone of half-angle $\alpha$ (see Figure 2(a)). Each ray within the cone has a power such that the beam intensity is uniform throughout the cone. In addition to using multiple rays in spherical geometry, each ray is allowed to refract (i.e., change angles) as it propagates through the plasma. The refraction is computed using a geometrical optics model with plasma refractive index being governed by local values of electron density. Figure 2(b) shows an example of rays propagating through a spherical plasma.

\section{Equation of State and Multigroup Opacity Models}

HELIOS-CR supports the use of: SESAME equation of state tables [5], PROPACEOS equation of state tables (see the Appendix), and an ideal gas equations of state. A bi-rational interpolation algorithm is used when computing EOS data at densities near solid density (where cohesive effects are important). HELIOS-CR also supports the use of PROPACEOS multi-group opacity tables. Displays of tabular data 
contained in EOS and opacity data files can be readily viewed while setting up simulations within the HELIOS-CR user interface.

\section{Zoning of Spatial Grid}

For a Lagrangian code, it is important to have a smooth distribution of zone masses. For a simulation with multiple materials, good mass-matching needs to be achieved not only within each material, but also at the interfaces. The automatic zoning algorithm in HELIOS-CR utilizes an iterative procedure to determine the optimum zoning. The procedure includes Fermi and parabolic algorithms. HELIOS-CR automatically determines which algorithm to use to minimize mass mismatch. The zoning can be readily adjusted and viewed when setting up a simulation.

\section{Time Step Control}

The time step size is determined using a set of stability and accuracy constraints. After each time step, the new time step is determined by:

$$
\Delta t^{\text {new }}=\operatorname{Min}\left(\frac{C_{k}}{\delta_{k}}\right), \quad k=1,6
$$

where $C_{k}$ is a user-specified constant (typically a few percent) for each stability constraint $k$, and:

$$
\begin{aligned}
& \delta_{1}=\operatorname{Max}\left(\frac{V_{j} P_{j}}{\Delta r_{j}}\right), \\
& \delta_{2}=\operatorname{Max}\left(\frac{1}{V_{j}} \frac{\Delta V_{j}}{\Delta t^{\text {old }}}\right), \\
& \delta_{3}=\operatorname{Max}\left(\frac{1}{\Delta E_{R, j}} \frac{\Delta E_{R, j}}{\Delta t^{\text {old }}}\right), \\
& \delta_{4}=\operatorname{Max}\left(\frac{1}{\Delta T_{i, j}} \frac{\Delta T_{i, j}}{\Delta t^{\text {old }}}\right) \\
& \delta_{5}=\operatorname{Max}\left(\frac{1}{\Delta T_{e, j}} \frac{\Delta T_{e, j}}{\Delta t^{\text {old }}}\right) \\
& \delta_{6}=\operatorname{Max}\left(\frac{1}{B_{j}} \frac{\Delta B_{j}}{\Delta t^{\text {old }}}\right)
\end{aligned}
$$


where the maximum values for $\delta_{k}$ are found by sweeping over all volume elements $j$. The first constraint is the Courant condition, while the other criteria constrain the fractional change of various physical quantities. In addition, global minimum and maximum time step sizes can be specified by the user.

\section{$\underline{\text { Energy Conservation }}$}

Energy conservation is monitored for the plasma at each time step. The energy partitioning among ion internal energy, electron internal energy, fluid kinetic energy, and radiation and magnetic fields, as well as energy gains and losses from the system (e.g., by laser deposition or escaping radiation) are written to a log file and can be graphically displayed using HydroPLOT. Energy conservation in most simulations is accurate to within $\sim 2$ to 3 percent.

\section{Graphical Output}

During a simulation, the temperature, density, pressure, and fluid velocity are dynamically displayed in a graphical progress monitor. This not only provides information on the status of the simulation, but also serves as an educational tool for students and junior scientists. At the end of a simulation, a wide variety of time-, space-, and frequency-dependent results can be easily displayed using HydroPLOT. This graphics tool supports displays of line plots, 2-D color contours, and 3-D surface plots. In addition, results from different HELIOS-CR simulations can be easily compared using the drag-anddrop capability in HydroPLOT.

\section{$\underline{\text { User Interface }}$}

HELIOS-CR has been designed to be intuitive and convenient to use, so that new users can quickly learn how to set up and run simulations. Problem setup involves cycling through a series of user interface (UI) panels. Online help for individual panels can be accessed directly through panel-specific Help buttons. An automated zoning capability allows users to easily view and adjust the spatial grid of the plasma. In addition, setup panels readily launch related applications for viewing data contained in EOS and opacity files, and for setting up customized atomic models for running non-LTE kinetics simulations. 


\section{$\underline{\text { Platforms }}$}

HELIOS-CR has been developed using cross-platform C++ user interface software, and utilizes the OpenGL [19] graphics application framework. This allows HELIOS-CR to run on a wide variety of platforms. HELIOS-CR currently runs on the platforms with the following operating systems: MS Windows (XP, NT, 95/98/2000), Macintosh OSX, and Linux.

\section{EXAMPLE APPLICATIONS}

To illustrate some of the uses of HELIOS-CR, we present example simulation results from radiatively-driven capsule implosions. These simulations are based on experiments performed at major laser and z-pinch facilities [20,21]. In each case, the collisional-radiative model was used to simulate the non-LTE atomic kinetics of the fuel dopants. Additional examples of HELIOS-CR simulations and benchmarks can be found elsewhere [22].

Figure 3 shows Lagrangian zone positions as a function of time computed for a z-pinch radiationdriven capsule implosion. The target is composed of DD fuel with a 0.3\% Ar dopant concentration, a $\mathrm{CH}$ ablator, and thin layers of PVA and Al. The capsule is embedded in a $14 \mathrm{mg} / \mathrm{cm}^{3} \mathrm{CH}_{2}$ foam. The plot shows the fuel reaching peak compression at $t \sim 21 \mathrm{~ns}$. Both the PVA layer and Al tracer layer are ablated radially outward by the radiation drive.

Figure 4 shows electron temperature and electron density color contour plots from a z-pinch radiation-driven capsule implosion in which the fuel contained both $\mathrm{Ar}$ and $\mathrm{Kr}$ dopants. The electron temperature in this simulation is seen to peak $T \sim 2.4 \mathrm{keV}$ at $t=21.1 \mathrm{~ns}$, while the electron density at the center of the implosion peaks at $t=22.3 \mathrm{~ns}$. The structure at the right $(r \gtrsim 0.17 \mathrm{~mm})$ at $t \sim 21-23 \mathrm{~ns}$ is due to the $\mathrm{CH}$ ablator.

Figures 5 and 6 show example results from a HELIOS-CR simulation of a laser-driven indirectdrive capsule implosion experiment performed at the OMEGA laser facility. Figure 6 shows the electron temperature and electron density profiles at a time near peak compression. Here, the temperatures at the 
center of the Ar-doped DD plasmas is seen to peak at $T \sim 1.2 \mathrm{keV}$, while the electron density in the fuel is $N_{e} \sim$ a few $\times 10^{23} \mathrm{~cm}^{-3}$.

Figure 6 shows the radiation flux escaping the capsule at a time near peak compression in the HELIOS-CR simulation. The radiation originates from the Ar dopant within the fuel. Note the detailed structure in the Ar spectrum due to Ar K-shell resonance lines and satellites. This shows the level of detail in the non-LTE atomic kinetics and radiation physics algorithms in HELIOS-CR is comparable to that found in high-quality spectral analysis codes or spectral post-processors.

\section{SUMMARY}

HELIOS-CR is an easy-to-use 1-D radiation-hydrodynamics code that simulates the dynamic evolution of plasmas in HEDP experiments. It includes sophisticated physics algorithms for simulating in detail the physical processes that affect LTE and non-LTE plasma radiative properties. It is an effective design and analysis tool for experimentalists involved in the study of laser-produced plasmas, radiativelyheated plasmas, and discharge-produced plasmas. Because of its ease of use and supporting graphics, it is particularly useful for training graduate and undergraduate students in the area of plasma and radiation physics. 


\section{APPENDIX A}

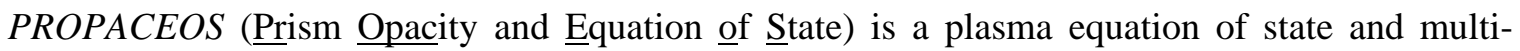
frequency opacity code used in the study of HEDP plasmas. It utilizes the same atomic and radiation physics algorithms as those described above for HELIOS-CR (see section on Atomic Kinetics Modeling). In most instances, EOS and opacity tables are computed for plasmas assumed to be in LTE, in which case atomic level populations are obtained from Boltzmann statistics and the Saha equation. However, PROPACEOS also has the capability to generate tables for non-LTE plasmas. In this case, atomic level populations are computed based on the steady-state solution of atomic rate equations, with contributions from photoionization and photoexcitation neglected (these are neglected because the radiation field, being nonlocal, is not a known quantity when generating the tabular data).

The bound-bound, bound-free, and free-free contributions to the opacity are computed using the same physics algorithms as those used in SPECT3D and HELIOS-CR, and are based on ATBASE photoionization cross sections and oscillator strengths. Group opacities are given by:

$$
\begin{gathered}
\sigma_{g}^{P A}=\frac{1}{\rho} \frac{\int_{x_{g}}^{x_{g+1}} d x B_{v}(T) \kappa_{v}}{\int_{x_{g}}^{x_{g+1}} d x B_{v}(T)} \\
\sigma_{g}^{P E}=\frac{1}{\rho} \frac{\int_{x_{g}}^{x_{g+1}} d x \eta_{v}}{\int_{x_{g}}^{x_{g+1}} d x B_{v}(T)} \\
\sigma_{g}^{R}=\frac{1}{\rho} \frac{\int_{x_{g}}^{x_{g+1}} d x\left(\frac{\partial B_{v}}{\partial T}\right)}{\int_{x_{g}}^{x_{g+1}} d x\left(\frac{\partial B_{v}}{\partial T}\right) \frac{1}{\kappa_{v}+s_{v}}}
\end{gathered}
$$

where $B_{v}(T)$ is the Planck function at temperature $T, \kappa_{v}$ is the absorption coefficient, $\eta_{v}$ is the emissivity, and $s_{v}$ is the scattering coefficient. Standard definitions for these quantities can be found in Mihalas [11]. For non-LTE plasmas, the Kirchoff relation $\left(\eta_{v}=\kappa_{v} B_{v}\right)$ does not hold, and $\sigma_{g}^{P E} \neq \sigma_{g}^{P A}$. It is important to note this distinction in non-LTE plasmas because the plasma emission (radiative cooling) rate is determined by $\eta_{v}$, while the plasma absorption (radiative heating) rate is determined by $\kappa_{v}$. 
The equation of state model in PROPACEOS includes contributions from: (1) translation of ions and elections, (2) electron degeneracy, (3) configuration effects from Coulomb interactions (DebyeHuckel correction), and (4) ionization and excitation. This model is expected to be reliable at densities below solid density ( $\rho \lesssim 10^{-1} \rho_{0}$ ), but does not currently include detailed physics for cohesive effects. A QEOS-type model [23] is currently under development for PROPACOES to model the near-solid density regime. For this reason, it is recommended that SESAME EOS tables be used when model plasmas near solid density.

Figure A.1 shows an example of frequency-dependent Planck group opacities computed for $\mathrm{CH}$ at temperatures of 10 and $60 \mathrm{eV}$ at $\rho=0.01 \mathrm{~g} / \mathrm{cm}^{3}$. Comparisons of PROPACOES results with TOPS data [24] have generally shown good agreement. 


\section{REFERENCES}

[1] Mihalas D, and Mihalas BW. Foundations in Radiation-Hydrodynamics. New York: Oxford University Press, 1984.

[2] Castor JI. Radiation Hydrodynamics. Cambridge, UK: Cambridge University Press, 2004.

[3] MacFarlane JJ. VISRAD - A 3-D view factor code and design tool for high-energy density physics experiments. J Quant Spectrosc Radiat Transfer 2002; 81: 287-300.

[4] MacFarlane JJ, Golovkin IE, Woodruff PR. SPECT3D Imaging and Spectral Analysis Suite. Technical report, PCS-R-045, Prism Computational Sciences, Madison, WI, 2003.

[5] Lyon SP, Johnson JD. SESAME: The Los Alamos National Laboratory Equation of State Database. Technical report, LA-UR-92-3407, Los Alamos National Laboratory, Los Alamos, NM, 1992.

[6] von Neumann J, Richtmyer RD. A method for the numerical calculation of hydrodynamic shocks. J Applied Phys 1950; 21: 232-237.

[7] Dresvin SV. Physics and Technology of Low Temperature Plasmas. Iowa State University Press, Ames, IA, 1977.

[8] Olson GL, Auer LH, Hall ML. Diffusion, P1, and other approximate forms of radiation transport. J Quant Spectrosc Radiat Transfer 2000; 64: 619-634.

[9] Levermore CD, Pomraning GC. A flux-limited diffusion theory. Astrophys J 1981; 248: 321-334.

[10] Olson GL, Kunasz PB. Short characteristic solution of the non-LTE line transfer problem by operator perturbation - I. The one-dimensional slab. J Quant Spectrosc Radiat Transfer 1987; 38: 325-336.

[11] Mihalas D. Stellar Atmospheres. New York: WH Freeman and Co, 1978.

[12] Hummer DG, Mihalas D. The equation of state for stellar envelopes. I. - an occupation probability formalism for the truncation of internal partition functions. Astrophys J 1988; 331: 794-814. 
[13] More RM. Applied Atomic Collision Physics, vol. 2. New York: Academic Press, 1982.

[14] Haynes DA. Private communication. 2001.

[15] Iglasius CA, DeWitt HE, Lebowitz JL, MacGowan D, Hubbard WB. Low-frequency electric microfield distributions in plasmas. Phys Rev A 1985; 31: 1698-1702.

[16] Wang P. Computation and application of atomic data for inertial confinement fusion plasmas. PhD dissertation, Dept of Nuclear Engineering and Engineering Physics, Univ of Wisconsin, Madison, WI, 1991.

[17] Chen FF. Introduction to plasma physics and controlled fusion. New York: Plenum Press, 1984.

[18] Duderstadt JJ, Moses GA. Inertial confinement fusion. New York: Wiley, 1982. p. 145.

[19] Woo M, Neider J, Davis T. OpenGL programming guide. Reading: Addison-Wesley, 1997.

[20] Bailey JE, et al. X-ray imaging measurements of capsule implosions driven by a z-pinch dynamic hohlraum. Phys Rev Lett 2002; 89: 095004-1 - 095004-4.

[21] MacFarlane JJ, et al. Dopant radiative cooling effects in indirect-drive capsule implosion experiments. Phys Rev E, submitted, 2004.

[22] Additional details on HELIOS-CR calculations can be found at http://www.prism-cs.com.

[23] More RM, Warren KH, Young DA, Zimmerman GB. Dopant A new quotidian equation of state (QEOS) for hot dense matter. Phys Plasmas 1988; 31: 3059-3078.

[24] Magee NH, Abdallah J, Clark REH, et al. Atomic structure calculations and new Los Alamos astrophysical opacities. In: Adelman SJ, Wiese WL, editors. Astrophysical applications of powerful new databases, vol. 78. Astronom Soc of Pacific, 1995. p. 51. 


\section{FIGURE CAPTIONS}

Figure 1: Flow diagram showing flow of data between HELIOS-CR and related applications and databases.

Figure 2. Schematic illustrations showing: (left) laser beam cone and target geometry for spherical plasma simulation, and (right) example of refraction of laser beam rays for a spherical target.

Figure 3. Lagrangian zone positions as a function of time from a HELIOS-CR simulation of a z-pinch radiation-driven capsule implosion experiment.

Figure 4. Electron temperature (top) and electron density (bottom) contour plots from a HELIOS-CR simulation of a z-pinch radiation-driven capsule implosion experiment with an Ar- and Kr-doped DD fuel.

Figure 5. Electron temperature and electron density profiles calculated for a laser-driven indirect-drive capsule implosion experiment.

Figure 6. Calculated frequency-dependent flux escaping an Ar-doped capsule at a time near peak compression, computed by HELIOS-CR in the simulation of a laser-driven indirect-drive capsule implosion experiment. The Ar K-shell resonance lines and their satellites are labeled.

Figure A.1. Frequency-dependent Planck group opacities for $\mathrm{CH}$ at $\rho=0.01 \mathrm{~g} / \mathrm{cm}^{3}$ and $T=10 \mathrm{eV}$ (dotted curve) and $T=60 \mathrm{eV}$ (solid curve) computed using PROPACEOS. 
Figure 1.

J. J. MacFarlane: "HELIOS-CR..."

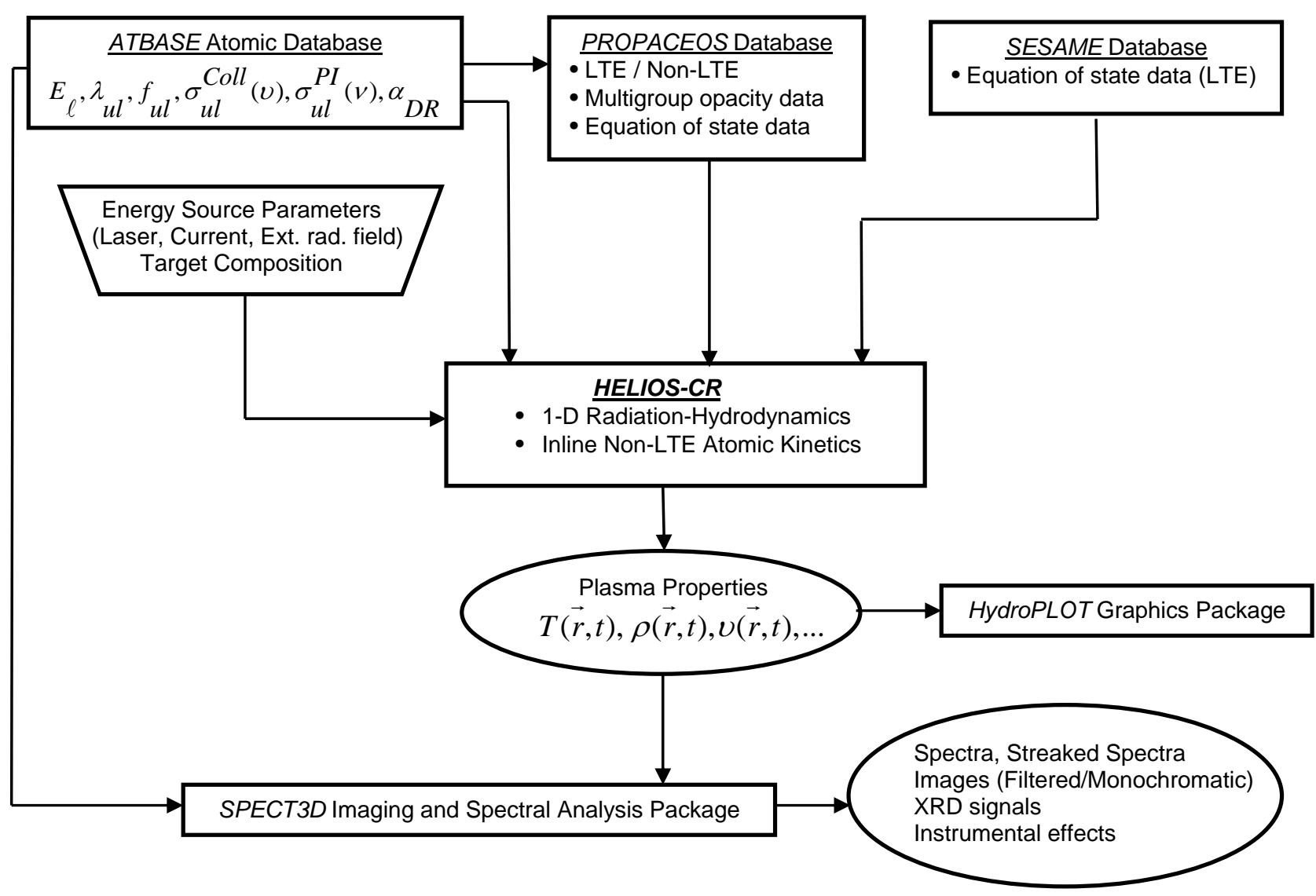


Figure 2.

J. J. MacFarlane: "HELIOS-CR..."

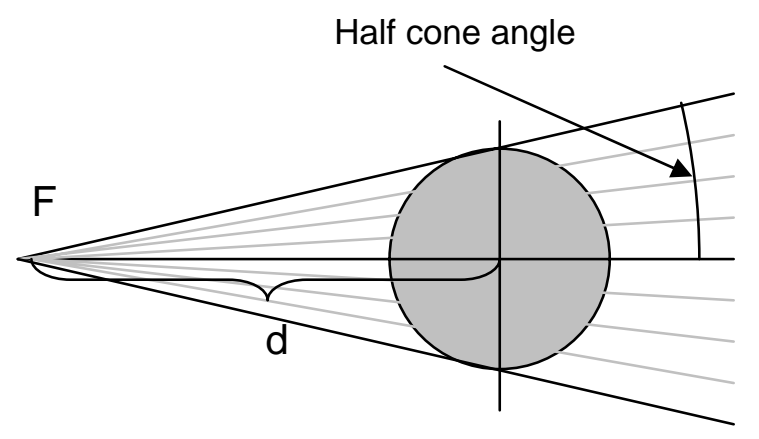

Equatorial plane

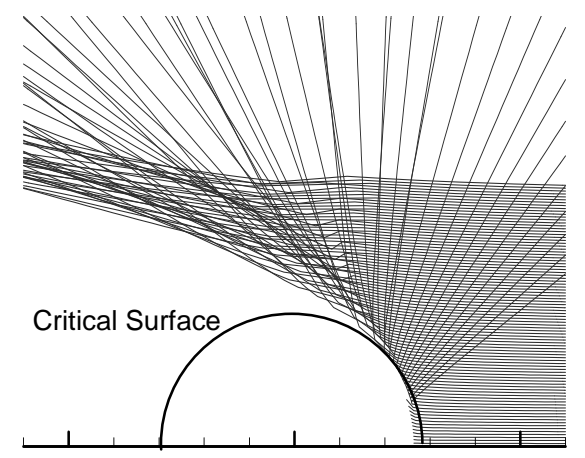

Radius 
Figure 3.

J. J. MacFarlane: "HELIOS-CR..."

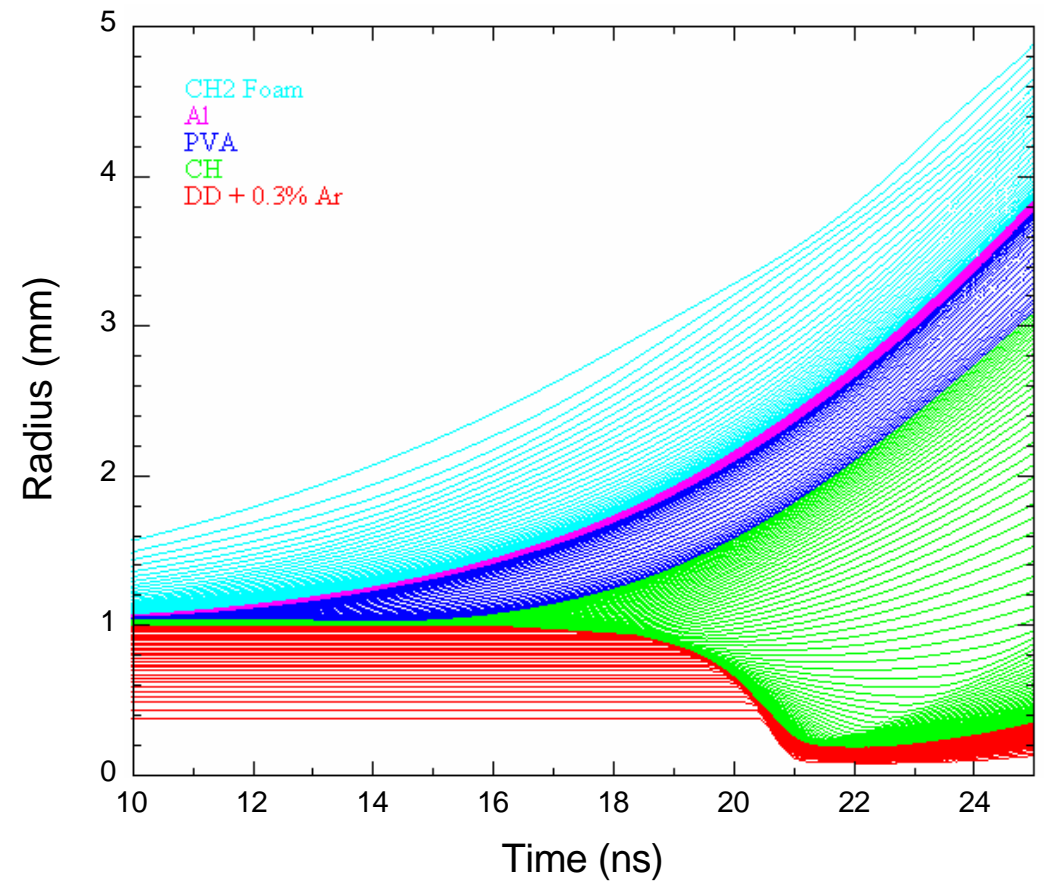


Figure 4.

J. J. MacFarlane: "HELIOS-CR...”
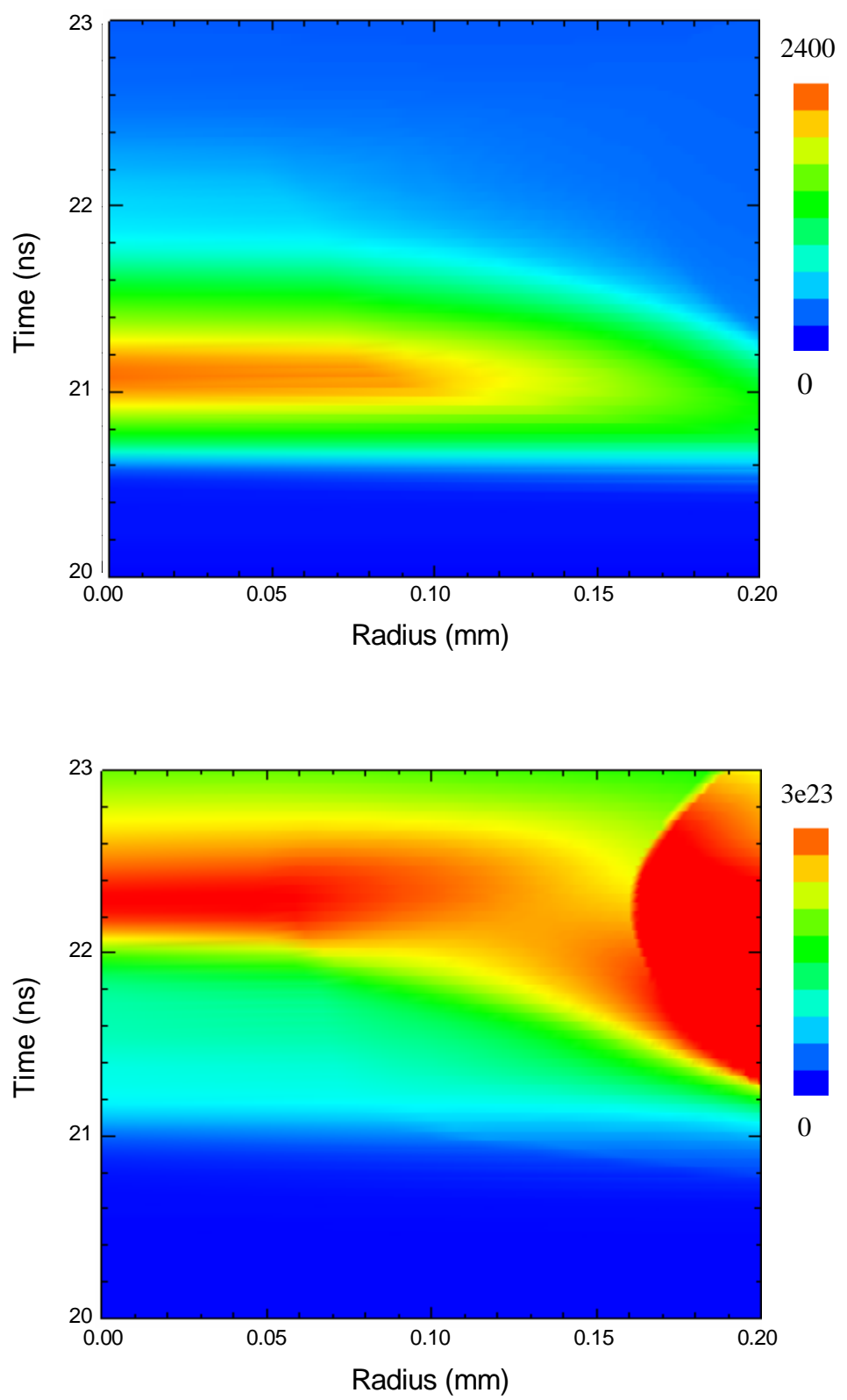
Figure 5.

J. J. MacFarlane: "HELIOS-CR..."

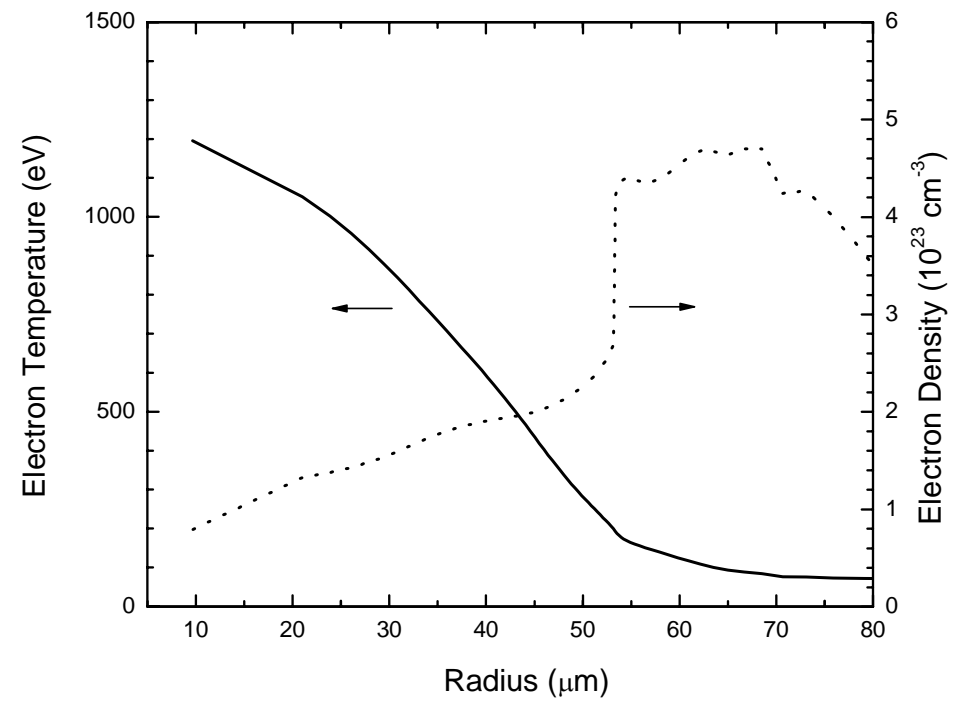


Figure 6.

J. J. MacFarlane: "HELIOS-CR..."

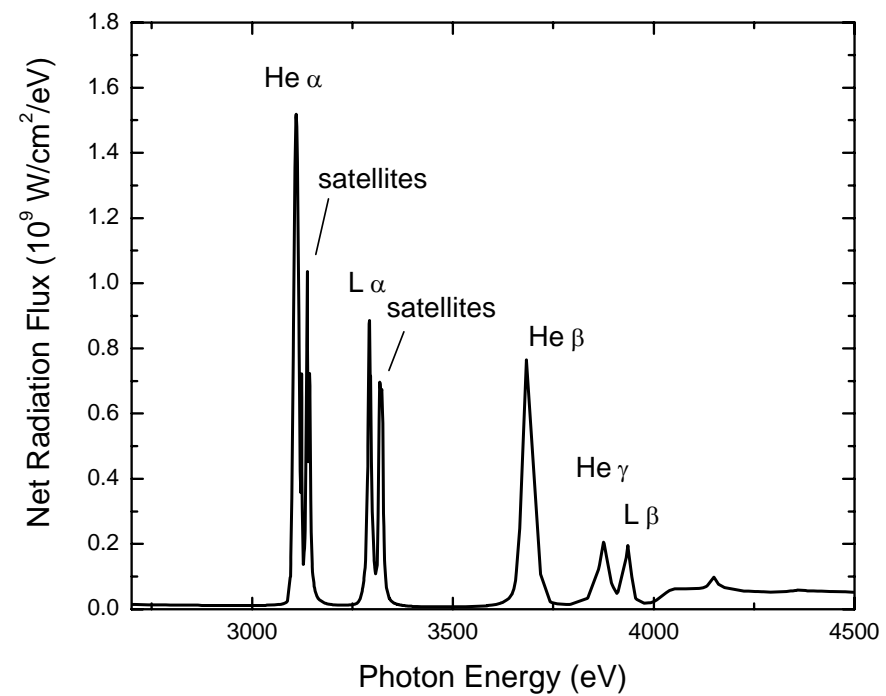


Figure A.1.

\section{J. J. MacFarlane: "HELIOS-CR..."}

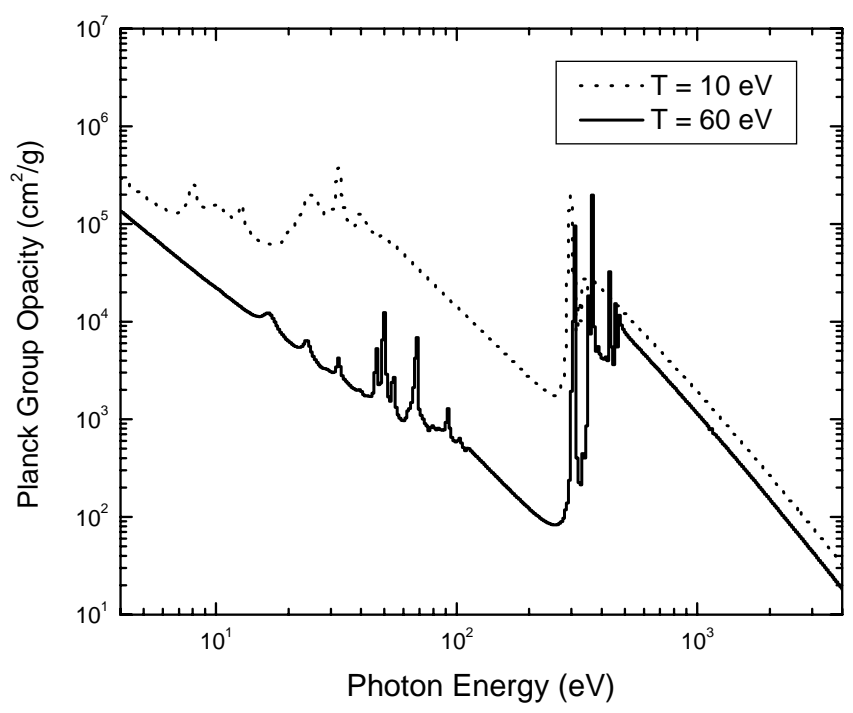

